\title{
Service User Perspectives of an Early Intervention in Psychosis Service: A Service Evaluation
}

\begin{tabular}{|r|l|}
\hline Journal: & Mental Health Review Journal \\
\hline Manuscript ID & MHRJ-09-2017-0037.R1 \\
\hline Manuscript Type: & Research Paper \\
\hline Keywords: & $\begin{array}{l}\text { Early Intervention Services, First episode of psychosis, Peer support, } \\
\text { Normalising, service user perspectives }\end{array}$ \\
\hline \multicolumn{2}{|c}{} \\
\hline
\end{tabular}

\section{SCHOLARONE ${ }^{m}$ \\ Manuscripts}




\begin{abstract}
Introduction: Early Intervention in Psychosis (EIP) services are recommended for people who experience a first episode of psychosis in the UK. Further research to understand the experience of people using EIP services is needed in order to improve these services. Purpose: This study aimed to understand SUs' perspectives of an EIP service. It was conducted as part of a service evaluation which aimed to identify areas for service improvement Method: Individual interviews $(n=9)$ and one focus group $(n=7)$ were conducted. Discussions focused on open questions pertaining to Service Users' (SUs) experiences of accessing the EIP service. Results: Inductive thematic analysis was used and three main themes were generated; Consistency and Communication, facilitating therapeutic relationships between EIP service staff and SU's. Pushing Boundaries, relating to the importance of services taking a graded approach to developing therapeutic relationships and (re)engaging in activities; and Normalising and Validating experiences of psychosis. Discussion: Participants emphasised the importance of relationships with EIP service staff and fellow SUs and highlighted how SUs can feel fearful and vulnerable when staff are not accessible or they view their care as inconsistent. Participants further emphasised the need for practitioners to balance an approach that de-stigmatises psychotic experiences whilst validating distress. Implications for Practice: Consistency of support from EIP services can be as important as flexibility. Clinicians should carefully consider the balance between validating and normalising distressing experiences associated with Psychosis. Offering social activities with other SUs can facilitate therapeutic relationships and recovery but the results suggest that this should be facilitated in a graded way.
\end{abstract}

Keywords: Early Intervention in Psychosis services, first episode of psychosis, peer support, normalising, service user perspectives 


\section{Introduction}

Early Intervention in Psychosis (EIP) services are specialist multi-disciplinary community mental health teams, recommended for people who experience a 'first episode of psychosis' (Department of Health, 1999; NICE, 2002; NICE, 2014). These services were introduced in the UK following research highlighting carer and Service User dissatisfaction with mental health services, but also the association between the duration of untreated psychosis and poorer prognosis (Tanskanen, et al., 2011; Lester, 2012). Individuals experiencing a first episode of psychosis can face barriers in obtaining prompt access to services. This has included worry about the stigma associated with mental illness, resulting in help often not being sought until crisis point (Tanskanen, et al., 2011).

In comparison to Community Mental Health Teams, EIP services often have smaller caseloads allowing for a more assertive and flexible approach. EIP services offer a youth friendly service delivery, with a focus on social inclusion and therapeutic optimism (British Psychological Society, 2005; Jones, et al., 2010). Service Users (SUs) within EIP services are more likely to have fewer inpatient admissions, use medication for shorter periods, and have an improved prognosis and an increased chance of being in employment (British Psychological Society, 2014; Singh, 2010; Bird, et al., 2010; McGorry, 2015). Lester, et al. (2012) found SUs 'highly prized' EIP services, especially the frequency and flexibility of appointments and social interaction with other SUs. However, EIP services may set up unrealistic expectations that are difficult to replicate in other services (Lester, et al., 2012).

Mental Health Services must be judged on the experience of SUs as well as their outcomes (NICE, 2011). This is considered to be especially important in an EIP service due to the high level of contact and possible compulsory treatment in some cases. The UK government states that more should be done to improve SUs' experiences of NHS services in its White Paper; Equality and Excellence: Liberating the NHS (Department of Health, 2010). Understanding the lived experience of EIP service users enables concerns to be addressed and good practice identified. Overcoming apprehensions about EIP services should enable SUs to access services in a timely manner and make the best use of the interventions on offer.

Previous research (O'Toole, et al., 2004) used focus groups to explore the experience of 12 people using an EIP service. Practitioners' taking a 'human approach' with them was deemed a key facilitator of their recovery. Being involved with treatment decisions, the flexibility of the service, and a high Care Coordinator to SU ratio were also valued. Lester, et 
al. (2011) conducted a multi-site qualitative study in which three main themes were developed by the authors; relationships with key workers, the value of family support, and changing self-identity. Participants stated that developing a therapeutic relationship with EIP services was the most important factor. Engagement with EIP services could be compromised if appointments were experienced as too intensive. A sense of 'over engagement' seemed to be exacerbated by frequent changes in EIP service workers. Frequent appointments served as a reminder of one's 'illness' for participants who felt sufficiently 'recovered'. Being flexible with the frequency of appointments seemed to mitigate this problem. Participants valued support from their families and appreciated it when the EIP service collaborated with their family.

Harris, Collinson, and Das Nair (2012) conducted interviews with eight EIP service users and analysed the results using Interpretvie Phenemonological Analysis (IPA). The authors identified an overall theme of 'personal journey of recovery' and five superordinate themes of stigma, relationships, understanding the experiences, sense of agency, and impact on sense of self. Participants highlighted 'stigma', both internalised and from others, as a barrier to communication. 'Relationships' with professionals and peers helped to promote better 'understandings' and 'normalise' their experiences. Participants also reported that the EIP service helped to support their 'sense of agency' through a process of accepting their experiences, taking control of their care, and developing a more positive 'sense of self'. Barr, Ormrod, and Dudley (2015) used Q-Methodology to investigate what SUs found most valuable about EIP services and identified four key factors; the therapeutic relationship, medical care, psychological interventions and support, coping, and recovery. Relationships with EIP service workers were again identified as the most valued aspect of the service, with active listening, empathic validation, and consistency of care named as key aspects.

More recently, a meta synthesis conducted to explore how people experience EIP services (Hansen, Stige, \& Davidson, 2017) found five new and overarching themes a) Something is wrong, B) do it for myself, C) it's about people, D) a price to pay, and E) ongoing vulnerability. The study highlighted these themes as a process in which SUs go through in the contact with services. 


\section{Methods}

\section{Participant selection}

The service evaluation was advertised to current SUs of an EIP service through their Care Coordinator. The EIP service served both urban and rural populations. SUs who accessed the EIP service were aged between 14 and 35. A total of 16 SUs participated within either a focus group $(\mathrm{n}=7)$ or individual interviews $(\mathrm{n}=9)$.

\section{Data collection}

Interviews and the focus group were conducted by a Trainee Clinical Psychologist (S.W.) on placement with the EIP service. This evaluation was part of a larger service evaluation within the EIP service. Other aspects of the service evaluation, primarily using quantitative methods, enquired about experiences of specific components of the EIP service. The aspects of the evaluation reported in this paper aimed to freely explore individuals' experiences rather than enquire into specific areas of the service.

The broad question, "what has your experience of the EIP service been like?", was initially asked in all interviews to encourage participants to discuss any experiences they wished to share. Responses were then discussed in greater detail using exploratory questions such as "what in particular did you find useful, difficult, good, etc.?", encouraging statements to prompt participants to give more detail, and (in the focus group) comparative questions to support further reflection and discussion, such as "has anyone else had similar/different experiences?" and "what are other people's thoughts about that?". All interviews and focus groups were audio recorded and transcribed verbatim.

The focus group was held in a room at the local youth service building, where the EIP service co-facilitated an activity group. Focus groups have previously been used to study the experience of EIP service' clients (O'Toole, et al., 2004). They are thought to be an effective way of evaluating services as they help to facilitate detailed and rich conversations that explore different experiences (Breakwell \& Millward, 1995).

Interviews were also offered to SUs who preferred to share their views individually. Individual interviews took place in rooms at the EIP service base, youth service buildings, and the EIP service housing project. 


\section{Rigour and reflexivity}

One of the authors (S.W.) conducted the interviews and the focus group, transcribed the audio recordings, and analysed the transcripts to develop themes. Given the nature of the research design and analysis it is recognised that the author will bring their own experiences and values into the evaluation. To aid transparency some information is provided: author S.W. had been on placement as a Trainee Clinical Psychologist with the service, was a young white male, and was encouraged to think about mental health from a biopsychosocial perspective when working in the service. To promote rigour and reflexivity author C.S. also independently developed themes from the transcripts. Interpretations were then discussed with a third researcher (V.R.) who was not involved in the initial analysis. Different interpretations of the data were discussed and a consensus arrived at.

\section{Ethical approval}

All participants gave informed consent to participate. In line with the local NHS Trust policy, the service evaluation was approved by the Care Group Clinician's network before recruitment. The service evaluation proposal was also peer reviewed by the Department of Psychological Health and Wellbeing at the University of Hull.

\section{Data analysis}

Transcripts were analysed using Thematic Analysis based on the recommendations provided in Braun and Clarke (2006). Transcripts were initially read and re-read. Quotes from the transcripts which related to the research question were highlighted and considered. The quotes were then compared and contrasted for notable and reoccurring ideas, allowing the development of preliminary themes. Individual interviews and the focus group were initially analysed separately, compared with each other, and then grouped together. There were no notable differences between themes from the focus group and those from individual interviews. The paper aimed to provide a detailed account of themes which were pertinent to the EIP service and were endorsed by many participants. Two themes were excluded from the final paper as they reflected the views of only a small minority of participants; pertaining to the wider context of the NHS (one participant) and use of medication (three participants). Author S.W. completed the transcribing, considered the initial quotes, and developed preliminary themes. Authors S.W., C.S., and V.R. developed and reviewed the themes, and produced the report. 


\section{Results}

Table 1: Overview of themes and the amount of participant endorsement Consistency and communication

The majority of participants really valued the relationship with their Care Coordinators. Knowing that somebody was available for them to contact often made them feel more secure.

"[my Care Coordinator said] I'm there for you if you need to text or ring me like and I feel quite comfortable with that"

When Care Coordinators were absent or unavailable, participants reported an increase in distress. A breakdown in communication between Care Coordinators and participants seemed to mediate the experience of distress.

"Not being told that, my Care Coordinator is off due to illness or whatever until afterwards ... which caused me to have some problems"

Some participants really emphasised the importance of communication with their Care Coordinator and offered suggestions to help the EIP service overcome barriers; such as using online communication to provide information about who is available to contact.

"It would probably be better if they had some sort of web page or something, so that you get updates that you check"

"[Online communication would be helpful] say if you're off sick or stuff like that"

“When you're Care Coordinator is off, and you've got an online check if they're off or you can't get in contact with them"

The key role of the Care Coordinator in the EIP service was highlighted during the evaluation. Participants explained how they felt stuck without their Care Coordinator, which could lead to frustration. Some participants described how they often felt dependent upon one staff member who coordinated their care and support. They felt frustrated that nobody else in the team could support them.

"If [my Care Coordinator] wasn't available I'd be stuck for like three weeks upset and waiting to talk to someone ... I'm just like, that's really infuriating ... you have to go through your worker to do pretty much everything ... It's just a bit like urrrgh, why can't someone else help me", 
The consistency and continuity of care was really important. Although this varied for different participants at different times, the regularity and time between appointments were associated with distress. This highlighted a significant gap in the service whereby when a Care Coordinator was off work it appeared difficult to get appropriate cover. However as this is service specific it cannot be generalised to other EIP services. The opportunity to talk about the consistency of care was important for some. Providing this opportunity allowed some participants to communicate how they experienced their care and what they felt they needed. One participant highlighted that this conversation was in part facilitated by another staff member from the EIP service.

"I've found for a brief period of time that I didn't have enough consistent care ... and ... it really got to me ... and I started slipping back into how I was before I joined [The EIP service] ... then I just talked to my Care Coordinator and ... you know, and I talked to my psychologist and everything kind of got better after that"

It was not just relationships with staff members that SUs valued; they also highlighted the benefits of meeting other SUs and developing deep bonds. Participants reported that the regularity of contact with peers also facilitated their recovery journey.

"I've made some brilliant friends for life ... without it I'd be lost ... without these Wednesday sessions and the art class they do on a Tuesday ... without contact basically every week I'd be in a much worse place than I am now"

\section{Pushing boundaries}

The value of the EIP service supporting people back into 'life' was highlighted in the interviews. Participants described the importance of social activities helping them to improve their confidence and enter back into society. This seemed to involve a balancing act; being supported out of one's comfort zone whilst not being 'pushed over the edge'.

"It meant a lot ... to be able to get me to push my boundaries, get me out of my comfort zone so to speak"

"If people feel like they're being pushed too much that's another thing that could push them over the edge"

Participants valued a range of different activities offered by the service. It seemed as though going to 'new places' not only helped to build confidence, but also facilitated a sense of trust 
in their relationships with staff members. Developing trust by doing activities together helped some to feel more comfortable opening up about their difficulties.

"The team tend to get everyone involved with different activities, they try to invite you out and take you to new places to get you more open ... so that you've build trust with them so that you can tell them what's going on, because my big problem is that if I'd meet someone that I don't trust I'm not going to tell them anything"

"I think it's like arts and crafts courses and stuff like that. I think all these things kind of contribute towards ... making people comfortable enough to push the boundaries you know, building confidence ... I think all them things are crucial towards like... recovery in the long run"

Many participants valued being 'pushed' in a gradual way. It seemed as though small steps helped to build confidence slowly.

"They take them out a little bit more, like small steps, they try build ... build up slowly so that's been a good way of doing it"

"It was them little steps, them little steps towards big steps, and then bigger steps, and then before you know it, it doesn't even feel like a step anymore"

Although this process appeared slow, participants reported that being encouraged to move out of their comfort zone was one the most important interventions offered by the EIP service. They reflected on a sense of achievement after reaching the next step. By taking these steps, participants felt more willing to engage in new relationships.

"And it was pushing my boundaries ... for me to be able to push them boundaries ... obviously with [my Care Coordinator] ... in the long run ... did a hell of a lot better than anything else you know, it was those little confidence builders and then I took that, took that advice home ... pushed my boundaries, was scared as hell at first, but once I did it ... it felt like an achievement you know ... and then ... the next thing I did it come a bit easier, and the next thing I did come a bit easier, until where I can be here now talking with you".

"The courses that they offer and stuff like that ... being able to interact back in to society ... not like pushing you towards the deep end ... kind of like slowly ... dipping your toes in the water so to speak ... another way they kind of like slowly introducing you back in to ... towards a career and courses, things that'll help you become 
basically a productive member of society again ... without them courses I don't really think it would ... work that well you know without [The EIP service] offering them like courses"

It was not just the EIP service staff who helped participants to open up about their problems; hearing other SUs talk about their concerns encouraged these conversations too. Participants reported how beneficial they found hearing from other people who had travelled a similar path.

"Some people might not want to talk about what the thing is but like I think if you sort of encouraged it, not forced it but encouraged it, it would be beneficial for some people who wouldn't normally open up, like talking about ... if people who're in the session sort of talked about maybe what was on their mind, like if they're struggling I think it would help ... 'cos it's the perspective of someone who's going through it, not just the staff"

\section{Normalising and Validating}

The importance of feeling accepted and 'not stigmatised' was highlighted in some interviews. 'Chatting' at participants' homes about their problems in an open and informal way helped to normalise some of their experiences.

"They came to my house and like, I chatted with them for a bit, they made me feel a lot better about it 'cos like ... like even if I did have any of this stuff it's not like ... stigmatised here"

Meeting other people who accessed the EIP service on activities further helped to 'normalise' their experiences and foster a sense of connectedness with other people. Spending time with other EIP service users seemed to reduce both their fears about being judged and their sense of loneliness.

"It's nice to see other people with, like getting out and about as well, 'cos I think that helps as well, like go on the, on the days together. 'Cos like ... a lot of it is due to stigma ... you worry that other people will think of stuff about you".

"You sort of go to these sessions like you know that like it's not just you, like you're not on your own". 


\begin{abstract}
Although useful to some, attempts at 'normalising' people's symptoms or experiences appeared to cause some conflict in relationships between participants and EIP service staff. It seemed as though some participants felt that their experiences were minimised or invalidated.

"I've been told too many times in the last few weeks that I'm just having a bad day and it's a blip... and it's just like ... it's very frustrating because it's not just one bad day, it's a continuous bad day".
\end{abstract}

Honest feedback on their presentation and progress was really important for some participants. The perception that EIP service staff had 'sugar-coated' what they had said seemed to help in the short-term but led to problems later when participants felt that they were not doing as well as they initially thought. Participants reported that this change in perception and expectation set them back on their recovery journey and possibly led to a sense of disappointment.

“When I've been sugar-coated in sweetness ... I haven't had the honesty ... it's put me back a bit because I think I'm doing good ... in the long run I find actually I'm not doing good". 


\section{Discussion}

Focus groups and interviews provided rich information about the lived experiences of people accessing the EIP service. Three key themes were identified; 'Consistency and Communication', 'Normalising and Validating', and 'Pushing Boundaries'. All themes emphasised the importance of the relationships with either the EIP service staff or fellow SUs, which replicated the findings from previous research into the experience of EIP service users (Lester, et al., 2011; Harris, et al., 2012; Barr, Ormrod, \& Dudley, 2015). To the best of the authors' knowledge, this is the first EIP service evaluation to suggest, from the perspective of SUs, the potential value of taking a graded approach to building trust and pushing boundaries through activities and peer support in EIP services. Participants also provided an insight into the experience of interventions to de-stigmatise their psychosis and the potential to perceive that their distress in minimised.

\section{Consistency and communication}

The relationship between those offering and those using mental health services is essential for positive experiences (Farrelly \& Lester, 2014). Consistency and communication were highlighted by SUs as key factors in enabling therapeutic relationships with EIP service staff. Flexibility of support is a key principle of EIP services and has been shown to be highly valued by SUs (British Psychological Society, 2005; Lester, et al., 2012). Participants in this evaluation clearly valued this flexibility. However, they reported feeling vulnerable and fearful if Care Coordinators were not accessible, or they perceived that their care was inconsistent. The experience of consistency and containment can be understood from the perspective of attachment theory; with EIP service staff acting as attachment figures and activating 'attachment behaviour systems' (Bowlby, 1988). EIP service staff can provide a 'secure base' to support SUs in their recovery journey; although this may depend on the perception that staff are available and able to respond when called upon (Bowlby, 1988). Opportunities to communicate with workers seemed essential in providing containment or reassurance. Discussing the experience of therapeutic relationships, as well as the frequency and consistency of appointments, may help to improve engagement and outcomes in EIP services.

\section{Pushing boundaries}


A graded process of supporting SUs to 'get back into life' and achieve their goals was another one of the most valued aspects of the EIP service, which is consistent with the value placed on promoting social inclusion in EIP services (The British Psychological Society, 2005; Jones, et al., 2010). Participants reported ideas consistent with theories of habituation or graded exposure (Grayson, Foa, \& Steketee, 1982) and the zone of proximal development (ZPD; Vygotsky, 1978). Achieving smaller goals gave SUs the confidence to face their next challenge. Goals that were perceived as out of their current ZPD were perceived as threatening and led to setbacks. Participants talked about disclosing their problems and discussing emotive topics in a graded way with EIP service workers and developing a trusting relationship was emphasised in facilitating this process. Engaging in social activities with both peers and professionals helped to facilitate trusting relationships and enabled SUs to talk about their problems. Engaging in reciprocal peer relationships has been shown to increase the sense of empowerment through the development of new ways of thinking and feeling (Resnick, et al., 2008).

\section{Normalising and validating}

Participants described finding benefit in low key or 'normalising' approaches. EIP services are recognised as taking an optimistic approach to recovery and a normalising approach whereby experiences of psychosis are viewed on a continuum as opposed to an 'all or nothing' view (e.g. normal or ill). This approach aims to reduce some of the distress associated with the stigma of psychosis and enhance recovery. Normalising approaches involve clinicians responding to SUs in a way that demonstrates empathy and understanding for the behavioural and emotional responses to a person's experiences (Lullman \& Lincoln, 2013). This approach attempts to validate these experiences and reduce distress. The current service evaluation highlighted instances whereby attempts to be optimistic about recovery or normalise experiences appeared to result in SUs feeling that their concerns or experiences were not taken seriously. Although different people vary in what they consider satisfactory validation, facilitating conversations about the experience or expectation of validation can improve therapeutic relationships (Leahy, 2008).

\section{Limitations of the evaluation}

Although this evaluation highlighted important considerations for services, caution should be taken when making generalisations due to the idiographic nature of the methodology. Future evaluations or research should consider facilitating more focus groups with different 
populations (Eliot \& Associates, 2005). Focus groups can be problematic as more confident people can dominate the conversation, resulting in a bias in the results. The selection of participants may also have been open to bias as the study was advertised through the EIP service activity groups and Care Coordinators. Participants who frequently attended the EIP service activities may have been more likely to experience them more positively than those who did not. Care Coordinators may also have selected SUs who they deemed 'good' for the evaluation, for example, SUs with whom they had a more positive relationship. Therefore, the results could represent a more positive experience of the EIP service. Further research could target SUs who do not engage with what the EIP service offers or develop positive relationships with Care Coordinators. It is further acknowledged that sample sizes within the evaluation were small, and the evaluation may have benefitted from the inclusion of a service user researcher to fully explore SU perspectives of the service. The evaluation may have also benefitted from further exploration regarding SUs' expectations of the service and what they hoped to gain from the support.

\section{Implications}

A number of tentative recommendations can be drawn from this service evaluation. This evaluation reaffirms the importance of relationships in EIP services; the 'small everyday things' as opposed to specific 'clinical interventions' (Topor, et al., 2018). Although flexibility is associated with positive outcomes in EIP services (Bird, et al., 2010), consistency appears to be just as important. EIP service staff should talk openly with SUs about the experience, frequency, content, and flexibly of appointments. This should be reviewed on a regular basis to avoid the risk of offering too much or too little support (Lester, et al., 2011). Talking openly about the possibility of breaks in support (e.g. holidays or sickness) and collaboratively agreeing a process to manage these times may also improve the experience of EIP service users. Recording these discussions in care plans may help to mitigate some of the problems associated with staff turnover. Offering an individually tailored and graded approach to the disclosure of problems and goals for intervention appears to be valued by SUs. Offering opportunities for EIP service staff and SUs to engage in activities can facilitate trust and engagement with the service. Finally, clinicians should reflect on the language they use in conversations and carefully consider the balance between empathising, validating, and normalising the experiences of people using EIP Services.

\section{Future evaluation or research}


Supporting users of EIP services to co-develop and co-facilitate future evaluations may enable future evaluations to ask questions that are more meaningful to those accessing the service. Providing education to EIP service workers on attachment theory in order to appreciate the role consistency and the experience of breaks could be evaluated to see if this influences the experiences of both EIP service users and workers. 


\section{References}

Barr, K., Ormrod, J. \& Dudley, R., 2015. An exploration of what service users value about early intervention in psychosis services. Psychology and Psychotherapy: Theory, Research and Practice, Volume 88, pp. 468-480.

Bird, V. Premkumar, P. Kendall, T. Whittington, C. Mitchell, J. Kuipers, E. (2010). Early intervention services, cognitive-behavioural therapy and family intervention in early psychosis: systematic review.. British Journal of Psychiatry . 197 (5), 350-356.

Bowlby, J. 1988. A secure base. 2 ed. New York : Routledge .

Braun, V. \& Clarke, V., 2006. Using thematic analysis. Qualitative research in psychology, 3(2), pp. 77-101.

Breakwell, G. M. \& Millward, L. J., 1995. Basic Evaluation Methods: Assessing Process, Practice and Procedure. Leicester: BPS Books .

Department of Health, 2010. Equity and Excellence: Liberating the NHS. White Paper. [Online]

Available

at: http://www.dh.gov.uk/en/Publicationsandstatistics/Publications/PublicationsPolicyAndGuida nce/DH_117353

Eliot \& Associates, 2005. How to conduct a focus group. [Online] Available https://assessment.trinity.duke.edu/documents/How to Conduct_a_Focus_Group.pdf [Accessed 2 February 2017].

Farrelly. S. \&. Lester, H. 2014. Therapeutic relationships between mental health service users with psychotic disorders and their clinicians: a critical interpretive synthesis.. Health and Social care in the community, 22(5), pp. 449-460.

Grayson JB, Foa, E. \&. Steketee, G.S. 1982. Habituation during exposure treatment: Distraction. behaviour research and therapy, Volume 20, pp. 323-328.

Hansen H, Stige SH \& Davidson L. (2017). How Do People Experience Early Intervention Services for Psychosis? A Meta-Synthesis. Qualitative health research . 28 (2).

Harris, K., Collinson, C. \& Das Nair, R., 2012. Service-users' experiences of an early intervention in psychosis service: An interpretative phenomenological analysis. Psychology and Psychotherapy: Theory, Research and Practice, Volume 85, pp. 456-469.

Jones, P., Shiers, D. \& Smith, J., 2010. IRIS Early Intervention in Psychosis. HEAD TO HEAD Specialist EIP services versus a CMHT model for providing early intervention in psychosis. Information for service planners \& commissioners. [Online] Available at: http://www.iris-initiative.org.uk/silo/files/head-to-head--eip-services-versus- 
cmhts--guidance-for-commissioners-and-service-planners.pdf

[Accessed 1803 2018].

Leahy, R. . (2008). The Therapeutic Relationship in Cognitive-Behavioral Therapy. Behavioural and cognitive Psychotherapy. 36 (6), 769-777.

Lester, H. Marshal, M. Jones, P. Fowler, D. Amos, T. Khan, N \& Birchwood, M. 2011. Views of Young People in Early Intervention Services for First-Episode Psychosis in England. Psychiatric Services, 62(8), pp. 882-887.

Lester, H. et al., 2012. Service users' views of moving on from early intervention services for psychosis: a longitudinal qualitative study in primary care.. British Journal of General Practice, 62(596), pp. 183-190.

Lüllmann E., Lincoln T. M. (2013). The effect of an educating versus normalizing approach on treatment motivation in patients presenting with delusions: an experimental investigation with analogue patients. Schizophr. Res. Treat. 2013.

McGorry, P. D., 2015. Early intervention in psychosis: Obvious, effective, overdue. Journal of Nervous and Mental Disease, 203(5), pp. 310-318.

NICE, 2011. Service user experience in adult mental health: Improving the experience of care for people using adult NHS mental health services, s.1.: National Collaborating Centre for Mental Health commissioned by the National Institute for Health and Clinical Excellence

O'Toole, M. S, 2004. Treating first episode psychosis - the service users' perspective: a focus group evaluation. Journal of Psychiatric and Mental Health Nursing, Issue 11, pp. 319-326.

Resnick S.G \& Rosenheck, R.A. 2008. Integrating Peer-Provided Services: A Quasi experimental Study of Recovery Orientation, Confidence, and Empowerment. Psychiatric services , 59(11), pp. 1307-1317.

Singh, S. P., 2010. Early intervention in psychosis. The British Journal of Psychiatry, 196(5), pp. 343-345.

Tanskanen S, Morant N, Hinton M, Lloyd-Evans B, Crosby M, Kilapsy H, Raine R, Pilling S $\&$ Johnson S. . (2011). Service user and carer experiences of seeking help for a first episode of psychosis: a UK qualitative study. BMC Psychiatry. 11 (157),

The British Psychological Society, 2005. Early Intervention in Psychosis Services. The role Clinical Psychologists can plan. Division of Clinical Psycholoy Psychosis and Complex Mental Health Faculty Briefing Paper No. 20, Leicester: The British Psychological Society.

The British Psychological Society, 2014. Understanding Psychosis and Schizophrenia, Leicester: The British Psychological Society. 
Topor A, Boe TD \& Larsen IB. (2018). Small things, micro-affirmations and helpful professionals everyday recovery-oriented practices according to persons with mental health problems. Community Mental Health Journal . PP 1-9.

Vygotsky, L., 1978. interaction between learning and development. In: mind and society . Cambridge MA: Harvard university press, pp. 79-91. 
Table 1.

\begin{tabular}{ll}
\hline Theme & $\begin{array}{l}\text { Amount of Participants } \\
\text { Endorsing Theme }\end{array}$ \\
\hline Consistency and Communication & $8(50 \%)$ \\
Pushing Boundaries & $5(31 \%)$ \\
Validating and Normalising & $8(50 \%)$ \\
\hline
\end{tabular}

